\title{
SERO'TONIN AND OCTOPAMINE HAVE OPPOSITE MODULATORY EFFECTS ON THE CRAYFISH'S LATERAL GIANT ESCAPE REACTION $^{1}$
}

\author{
DAVID L. GLANZMAN AND FRANKLIN B. KRASNE ${ }^{2}$ \\ Department of Psychology, University of California at Los Angeles, Los Angeles, California 90024
}

Received July 12, 1982; Revised April 29, 1983; Accepted May 25, 1983

\begin{abstract}
Serotonin and octopamine have opposite effects on a simple behavioral response, the crayfish's lateral giant escape reaction. Specifically, serotonin depresses the lateral giants' responsiveness, whereas octopamine enhances it. Both effects are largely confined to the disynaptic pathway from the sensory afferents to the lateral giants, although, occasionally, small effects are also seen in the monosynaptic pathway to the lateral giants. One specific locus of the octopaminergic effect is the synapse between the afferents and the largest of the sensory interneurons in the disynaptic pathway, interneuron A. Serotonin, however, does not have a consistent effect at this synapse. Although serotonin and octopamine modulate the excitability of the escape response, neither monoamine appears to significantly alter the rate at which this response habituates.
\end{abstract}

Much attention has recently been given to the modulatory actions of various monoamines on synaptic transmission in the central nervous system and to the possible behavioral roles of these actions. At present, however, the only instance where a direct relationship can be seen between a monoamine's effect on a neuron and its effect on a behavior is in an invertebrate: serotonin causes transmission between the primary afferents and the gill withdrawal motoneurons of Aplysia to be presynaptically facilitated; this presynaptic facilitation in turn mediates behavioral dishabituation of Aplysia's gill withdrawal reflex (Kandel et al., 1981). Although there are now many other instances, both for vertebrates and invertebrates, where monoamines are known to affect behavior (e.g., Ungerstedt, 1979; Davis et al., 1980; Livingstone et al., 1980; Gelperin, 1981; Kravitz et al., 1981; Kupfermann and Weiss, 1981; Willard, 1981; Horvitz et al., 1982), in none of these instances can precise connections between synaptic and behavioral effects yet be drawn. However,

\footnotetext{
${ }^{1}$ This research was supported by United States Public Health Service Grant NS 06487 and a Grass Foundation Fellowship to D. L. G., and by United States Public Health Service Grant NS 08108 to F. B. K. Part of this research was carried out at the Marine Biological Laboratory, Woods Hole, MA. We thank Barbara Beltz and Edward A. Kravitz for making the results of their serotonin antibody work on lobster known to us prior to publication. We also thank Jeffrey J. Wine for helpful comments and Carl Higgins for typing the manuscript.

${ }^{2}$ To whom reprint requests should be addressed, at Department of Psychology, University of California, 405 Hilgard Avenue, Los Angeles, CA 90024 .
}

if we are to understand the natural functions of monoamines in nervous systems and of the modulatory types of synaptic actions which these substances often seem to mediate, we must develop a range of preparations in which the behavioral roles of central monoaminergic actions can be studied. With this goal in mind, we investigated the effects of two monoamines, serotonin $(5-\mathrm{HT})$ and octopamine, on the lateral giant (LG) escape reflex of the crayfish. The neural circuitry underlying this behavior has been exceptionally well analyzed (Krasne, 1969; Zucker, 1972a, b, c; Krasne and Wine, 1977; see below); thus, the relationship between the behavioral and neurophysiological effects of the monoamines, if any, should be easy to determine. Serotonin and octopamine seemed like promising candidates for study because they are already known to have both peripheral and central effects in crayfish (Grundfest and Reuben, 1961; Dudel, 1965; Livingstone et al., 1980; Jacobs and Atwood, 1981). Moreover, many of the known effects of octopamine in arthropods have led to the suggestion that octopamine may be the arthropod analogue of epinephrine (Battelle and Kravitz, 1978; Candy, 1978; Evans and O'Shea, 1978; Hoyle and Dagan, 1978; O'Shea and Evans, 1979; Jacobs and Atwood, 1981). If this were so, one might well expect octopamine to affect a "flight" response like the crayfish's escape reaction.

\section{Materials and Methods}

Procambarus clarkii, measuring 8 to $10 \mathrm{~cm}$ from rostrum to telson, were used in these experiments. The 
monoamines were injected directly into the abdominal nerve cord's circulatory system via cannulation of the ventral artery. Prior to cannulation, crayfish were cooled to approximately $5^{\circ} \mathrm{C}$ in dechlorinated tap water and their chelipeds were autotomized. In order to prevent clotting, an animal's blood was first replaced with cold, aerated crayfish saline (A. Kramer, personal communication). The animal was then pinned out dorsal side up in a Sylgard-lined dish, and a dorsal dissection for cannulation of the ventral artery and recording from the LGs was performed (Brown and Sherwood, 1981). The cannula was a blunted 23 gauge hypodermic needle joined to a length of polyethylene tubing (inside diameter, 0.023 inch; outside diameter, 0.038 inch) which was attached to a gravity-fed solution administration apparatus (Travenol Minidrip). The cannula was inserted into the descending artery (which feeds directly into the ventral artery) through a small incision in the bulbous arteriosis and secured with a fine thread of surgical silk. The perfusate flowed at a rate of approximately $50 \mathrm{ml} / \mathrm{hr}$. A three-way stopcock (Becton-Dickinson, No. 3157, or Hamiton IIV valve, No. 8673) permitted the experimenter to switch rapidly between perfusing solutions. The experimental solutions were normal crayfish saline (Van Harreveld, 1936) and saline containing one of the monoamines, either DL-octopamine hydrochloride (Sigma Chemical Co.) or 5-HT creatinine sulfate (Sigma). Early experiments were carried out with bicarbonate-buffered saline like that used in previous work on the crayfish's escape system in this laboratory. In later experiments, $10 \mathrm{~mm}$ Trizma or $20 \mathrm{~mm}$ HEPES, with $\mathrm{pH}$ adjusted to 7.3 and isotonicity maintained by reducing the $\mathrm{Na}^{+}$concentration of the saline, were used. (HEPES gave the superior buffering.) For the most part, all three buffers gave the same results. However, transmission from the sensory afferents to interneuron A (see below) appeared to be depressed at the decreased levels of $\mathrm{pH}$ which develop in bicarbonate-buffered 5-HT solutions. Therefore, the 5-HT experiments reported on this interneuron were all done with Trizma or HEPES buffer.

During experiments the animal was immersed in circulating, aerated saline kept at 12 to $16^{\circ} \mathrm{C}$. The perfusing solutions were also aerated but were kept at room temperature.

Before recording, the abdominal nerve cord was severed, usually at the connective between the first and second abdominal ganglia, to prevent known descending inhibition (Krasne and Wine, 1975) or other possible descending effects from interfering wth the LGs' excitability. During this operation, care was taken not to damage the underlying ventral artery. Also, the abdominal roots innervating the phasic flexor muscles were cut to prevent muscular twitching. The LGs were impaled with intracellular electrodes just rostral to the septal junction in either the third or fourth abdominal ganglion. The electrodes contained $3 \mathrm{M} \mathrm{KCl}$ and had impedances of 5 to 20 megohms. Hook electrodes were used to stimulate the second root of the same ganglion in which the LGs were penetrated, ipsilateral to the penetration. In certain experiments (described under "Results") wire electrodes were placed on the cord's ventral surface to record the action potential of an identified sensory in- terneuron, interneuron $A$, and this interneuron's afferent input was stimulated via hook electrodes on the second, third, and fourth roots of the last abdominal ganglion.

The general experimental protocol was as follows. Shocks were delivered to second roots in blocks of 10 , at rates of $1 / 5 \mathrm{sec}$ to $1 / 60 \mathrm{sec}$ alternating with 3 -min rest periods. At the end of some stimulus blocks the perfusate was switched from normal saline to monoamine-containing saline, or vice versa. The size of the evoked EPSP in monoamine-containing saline could thus be compared to that in normal saline for a stimulus of fixed intensity delivered on an unchanging schedule. The data presented below are all based upon comparisons between drug and control conditions in the same preparations.

\section{Results}

The afferent side of the neuronal circuitry underlying the LG escape response is shown, somewhat simplified, in Figure $1 A$. Brief shocks delivered to axons of sensory hair afferents on the dorsal surface of the crayfish's abdomen give rise to a compound EPSP in the LGs (Krasne, 1969). The first two components of this EPSP, termed $\alpha$ and $\beta$, are illustrated in Figure $1 B$. The $\alpha$ component is believed to arise from monosynaptic electrical input from the sensory afferents to the LGs. The $\beta$ component is believed to arise from disynaptic input to the LGs via a population of sensory interneurons (some of them identified) that receive chemical synaptic input from the primary afferents and make electrical synapses onto the LGs (Zucker, 1972a).

5-HT depresses LG EPSPs. When perfused through the crayfish's ventral artery in concentrations of $10^{-3}$ to $10^{-6} \mathrm{M}$ (lower concentrations have not yet been tried), 5 HT depressed the EPSPs evoked in the LGs by constant intensity second root shocks. This result is illustrated in
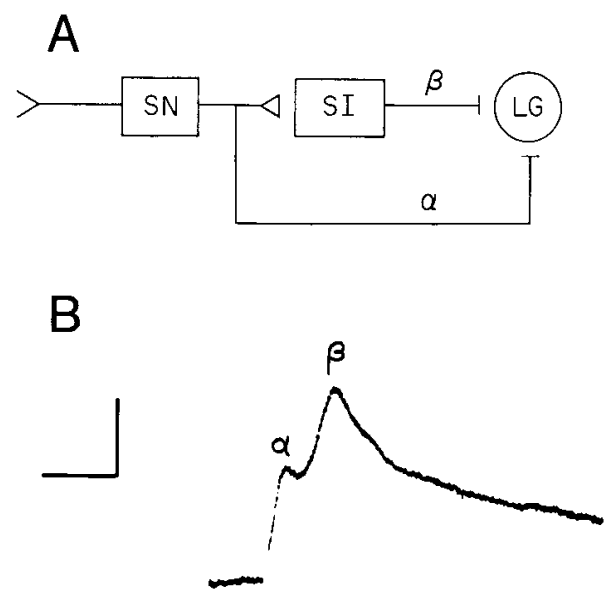

Figure 1. A, Schematic diagram of the neural circuitry underlying the lateral giant ( $L G$ ) escape response. The sensory neurons $(S N)$ form chemical synapses with the sensory interneurons $(S I)$ as well as direct electrical synapses with the LGs. The sensory interneurons also form electrical synapses with the LGs. The squares denote populations of neurons; the circle denotes an identified neuron. $B$, Record of a compound FPSP evoked in the LGs by stimulating the sensory neurons' axons. The contributions due to the monosynaptic and disynaptic pathways diagrammed in $A$ are labeled $\alpha$ and $\beta$, respectively. Calibration: horizontal, $4 \mathrm{msec}$; vertical, $4 \mathrm{mV}$. 
Figure 2. The LGs fired on the first and second trials during the control run in normal saline; by the 10th trial, the EPSP had declined to a subthreshold level. This decline reflects the normal habituation of the LG response (Krasne, 1969; Zucker, 1972b). During the 5-HT run, the EPSP was so depressed that the LGs failed to fire even on the first trial, and on subsequent trials, the EPSP amplitudes continued to decline. When the drug was washed out of the artery with normal saline, the LGs' responsiveness returned.

The time course of serotonin's effect on the LGs is illustrated in Figure 3. The amplitudes of the $\alpha$ and $\beta$ components of the EPSP have been graphed separately

$$
\text { CONTROL } \quad \text { WHT WASH }
$$

Trial

1<smiles>CCCCC</smiles><smiles>CCCC</smiles>

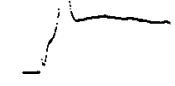

2
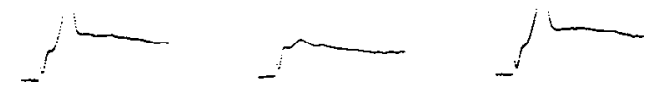

10
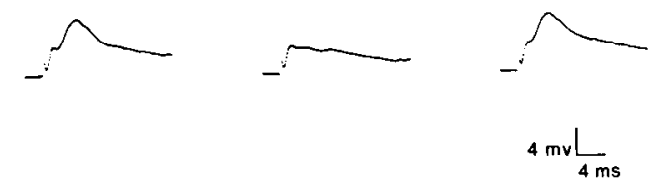

Figure 2. Effect of 5-HT on the LGs' responsiveness. The LGs were stimulated with second-root shocks of constant intensity at $1 / 5 \mathrm{sec}$ in blocks of 10 stimuli/hlock. Shown are the LGs' responses on the 1st, 2nd, and 10th trials of each block. During the Control and Wash trials, normal saline was perfused through the crayfish's ventral artery; during the $5-H T$ trials, $10^{-3} \mathrm{M}$ serotonin was perfused through the artery. On some trials the IGs gave a spike, which was truncated at the top of the oscilloscope screen. in this figure. Serotonin began to depress the LGs' excitability, as indicated by the decline in the $\beta$ component's amplitude, within $3 \mathrm{~min}$ after switching perfusates. With continued 5-HT perfusion, spiking in the LGs was completely suppressed, and $\beta$ 's overall amplitude declined further. After $20 \mathrm{~min}$ of washout with normal saline, the LGs recovered approximately their original level of excitability.

As Figure 3 indicates, 5-HT's major effect was upon the disynaptic $\beta$ component of the LGs' EPSP; the monosynaptic $\alpha$ component was frequently unaffected. In several such instances, 5-HT's lack of an effect on the $\alpha$ component was further verified by measurements of $\alpha$ 's size as a function of stimulus intensity (Fig. 4A). This lack of an effect on $\alpha$ rules out changes in the input impedance of the LGs or in the size of the afferent volley as a major cause of the serotonergic effect in such instances. Occasionally, however, 5-HT did depress $\alpha$ 's size, although the drug's effect on $\alpha$ was always much smaller than its effect on $\beta$. As described below, octopamine can also have small effects upon the size of the $\alpha$ component. When effects on $\alpha$ were seen, stimulusresponse plots such as that presented in Figure $4 B$ for octopamine indicated that the stimulus threshold for $\alpha$ did not change, whereas $\alpha$ 's amplitude with a supramaximal stimulus did. Therefore, the small changes in $\alpha$ which were occasionally seen were not due to changes in the spike threshold of the afferent fibers; rather, they reflected changes either in transmission between primary afferents and the LGs or in the LGs' input impedance.

Octopamine enhances LG EPSPs. Octopamine's effect upon the evoked EPSPs in the LGs was the opposite of 5-HT's: in concentrations of $10^{-3}$ to $10^{-6} \mathrm{M}$ (lower concentrations have not yet been used), ocotopamine enhanced the EPSPs' size. This result is illustrated in Figure 5. A stimulus which evoked only a subthreshold response from the LGs during the control run caused the LGs to spike during the octopamine run (trial 1). Moreover, on subsequent trials in octopamine, the EPSPs were all larger than on the corresponding control trials. Octopamine's enhancement of the LGs' excitability was

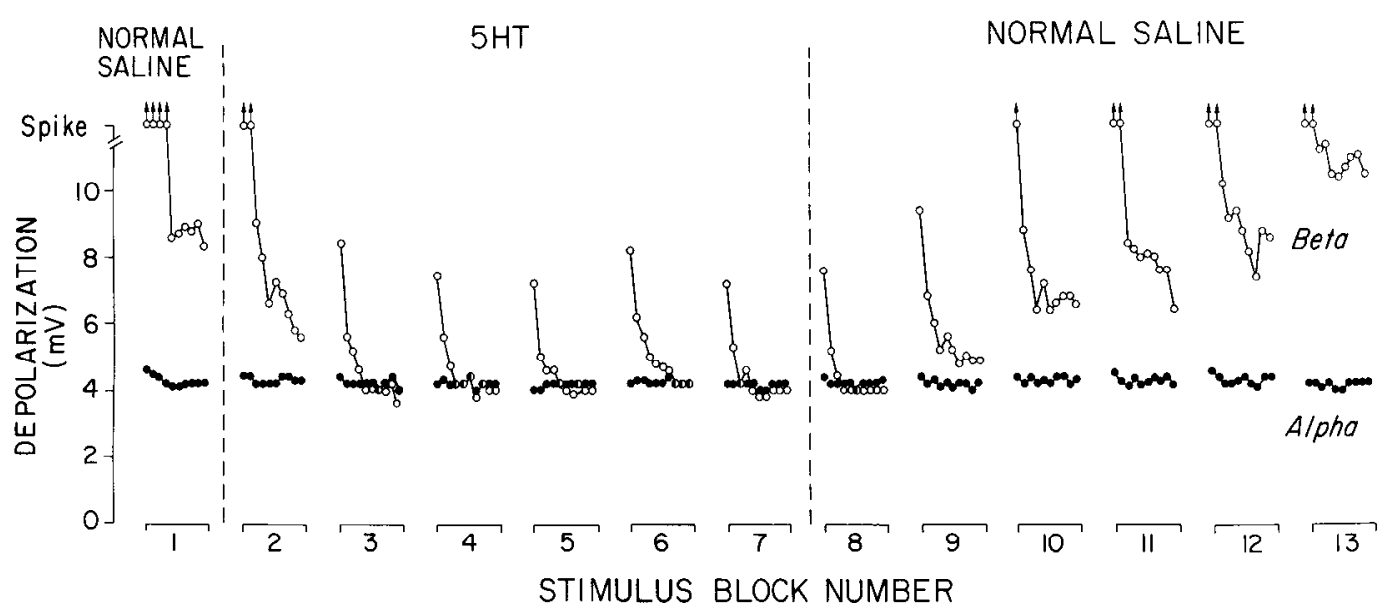

Figure 3. Time course of 5-HT's effect. In this graph, the amplitude of the $\alpha$ and $\beta$ components of the LGs' EPSP have been plotted over the course of a single experiment (the same experiment as that of Fig. 2). The data from all 10 trials of each stimulus block are shown. The solid circles represent the $\alpha$ component, the open circles represent the $\beta$ component. Arrows arising from certain of the $\beta$ symbols indicate the LGs fired on those trials. 
A

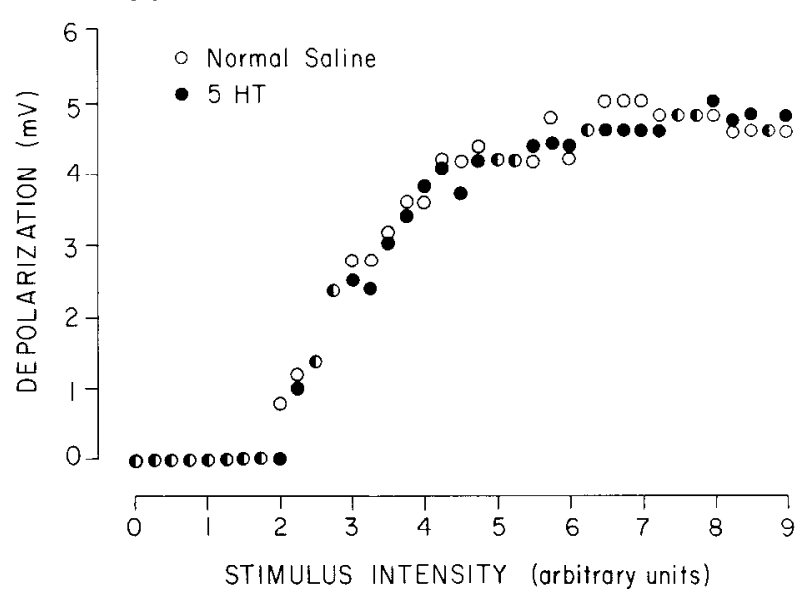

B

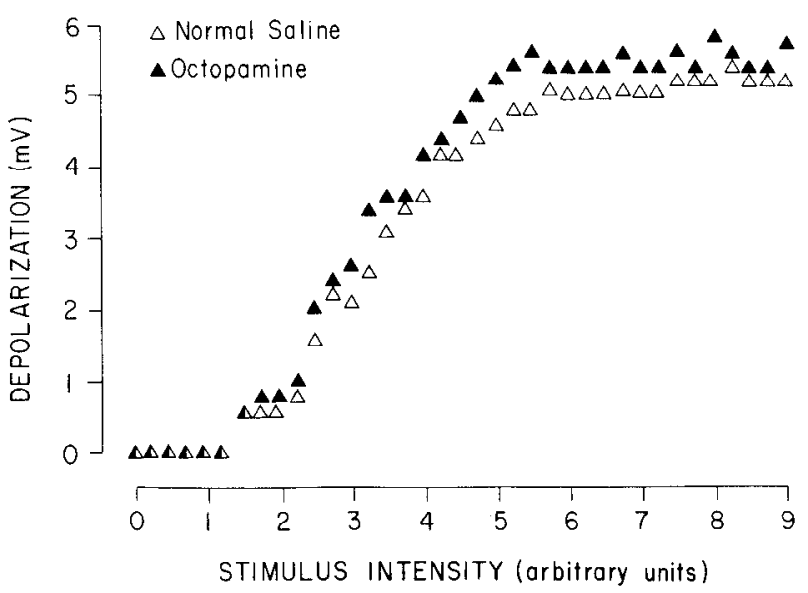

Figure 4. A, $\alpha$ component size as a function of stimulus intensity in 5-HT and normal saline. Second-root shocks were applied at $1 / 30$ sec. Open circles represent data from an experiment in which normal saline was perfused through the ventral artery; solid circles represent data from an experiment in which $5 \times 10^{-4}$ M 5 -HT was perfused through the artery. Both experiments were performed on the same preparation. 5 -HT had no effect on the amplitude of $\alpha$ at any stimulus intensity. $B, \alpha$ component size as a function of stimulus intensity in octopamine and normal saline. The protocol in these experiments was as above. The open symbuls represent data from a normal saline experiment; the solid symbols represent data from an experiment in which $5 \times 10^{-4} \mathrm{M}$ octopamine was perfused through the artery. Octopamine did not affect $\alpha$ 's threshold; it did, however, cause an increase in $\alpha$ 's size to suprathreshold, including supramaximal, shocks.

reversed when the perfusate was switched back to normal saline.

The time course of the octopaminergic effect is illustrated in Figure 6. In this experiment (different from the experiment of Fig. 5), the LGs fired on only the first trial of the control block. Within $3 \mathrm{~min}$ after switching to octopamine, the overall size of the $\beta$ component of the EPSP was enhanced. The LGs then began to respond to the stimuli with spikes, and by approximately $24 \mathrm{~min}$ after switching to octopamine, the LGs were spiking on almost every trial (stimulus block 7). When normal saline was reintroduced in the cannula, the LGs began to spike less frequently, and after $20 \mathrm{~min}$ they had returned approximately to their control level of excitability. As was the case for 5 -HT, octopamine's major effect was on the $\beta$ component of the EPSP. Occasionally, octopamine had a slight effect on the $\alpha$ component as well, as exemplified in Figure 6.

The monoamines do not significantly alter rate of habituation. When we inspected graphs of the monoamines' effects on the $\beta$ component of the EPSP, such as those of Figures 3 and 6, we thought it possible that 5-HT and octopamine might affect the rate at which the LGs' response habituated, in addition to its average amplitude. Two types of experiments were done to test this possibility. In both, the EPSPs were kept subthreshold so that we could measure the monoamines' effects on EPSP size throughout an experiment. In one type of experiment constant intensity stimuli were given and EPSP amplitudes were plotted as a percentage of the EPSP's size on the initial trial of each stimulus block. Thus analyzed, there were, at most, only minor differences in habituation between monoamine and control conditions. These experiments, however, left open the possibility that either a floor effect (in 5-HT's case) or a ceiling effect (in octopamine's case) might have limited the amount of habituation that could be seen. We therefore carried out a second series of experiments in which stimulus intensity was adjusted so that the size of the EPSP evoked on the first trial of each stimulus was kept approximately constant during saline and monoamine perfusion. There appeared to be no significant differences between the monoamine and control data in these experiments either.

Effect of the monoamines on the largest first-order sensory interneuron in the $L G$ circuit. The effects of 5HT and octopamine were largely limited to the disynaptic pathway between the sensory afferents and the LGs

$$
\text { CONTROL OCT WASH }
$$

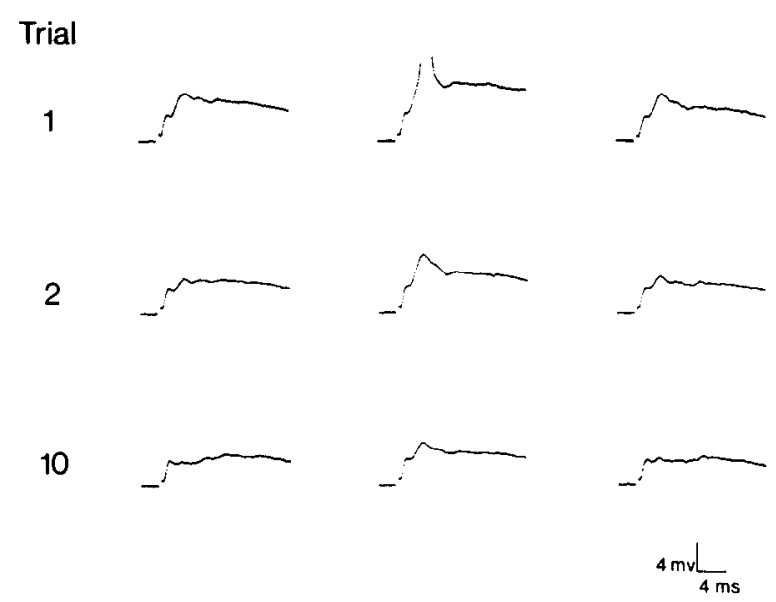

Figure 5. Effect of octopamine on the LGs' responsiveness. The LGs were stimulated with second-root shocks of constant intensity at $1 / 5$ sec. During the Control and Wash trials, normal saline was perfused through the ventral artery; during the Oct trials, $5 \times 10^{-4} \mathrm{M}$ octopamine was perfused through the artery. The truncated EPSP record for Trial 1 under octopamine represents an $L G$ spike. 


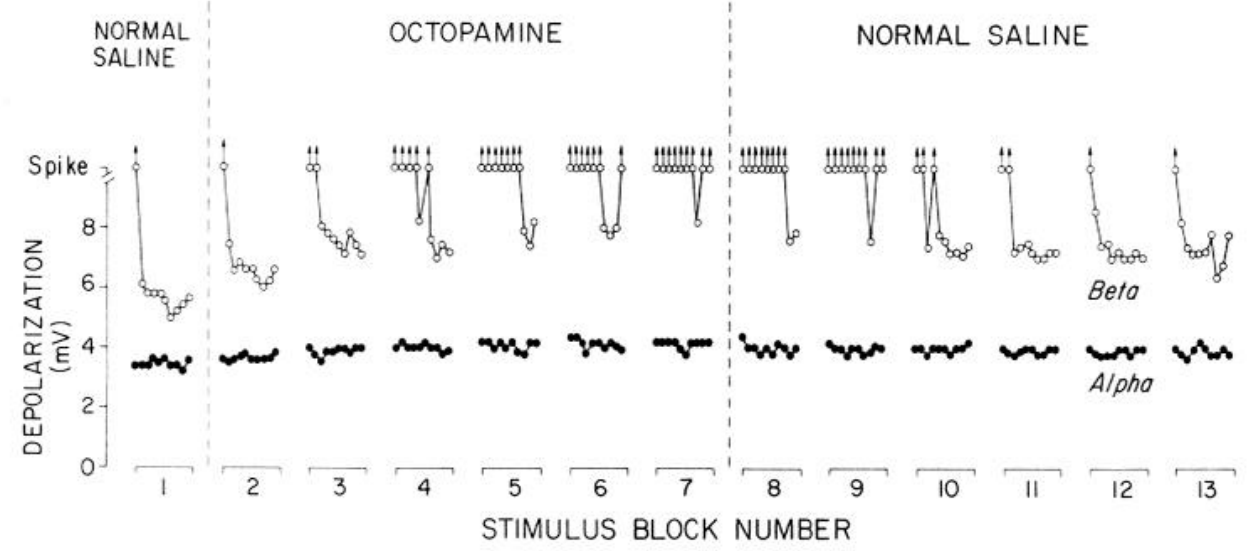

Figure 6. Time course of octopamine's effect. Conventions are those of Figure 3. These data are from an experiment in which $2 \times 10^{-3} \mathrm{M}$ octopamine was perfused through the ventral artery; otherwise the protocol was the same as in the experiment of Figure 2.

(Fig. 1A). The first synapse in this pathway, the chemical synapse between the afferents and the sensory interneurons, is the locus both of habituation of the escape reaction (Zucker, 1972b) and of inhibition that prevents undesirable effects of reafference during escape reactions (Bryan and Krasne, 1977a, b). It therefore seemed a likely locus for the monoamines' effects upon the $\beta$ component's size. Accordingly, we tested 5-HT's and octopamine's effects upon the size of the stimulus required to fire the largest, first-order sensory interneuron in the LG circuit, interneuron A (A) (Zucker, 1972a). Interneuron A's spike can be unambiguously identified in extracellular recordings from the ventral surface of the five to six abdominal connectives (Krasne and Lee, 1977). Axons of the sensory neurons which constitute $A$ 's afferent input were stimulated with single $0.1-\mathrm{msec}$ shocks at $1 / 30$ sec via hook electrodes placed under second, third, and fourth roots of the last abdominal ganglion (where $A$ 's cell body and dendrites are located). We determined the threshold for inducing $A$ to fire to this stimulus, as indicated by the appearance of $A$ 's extracellularly recorded spike, both in normal saline and in monoamine-containing saline. (For the interneuron A experiments, the concentrations of the monoamines were $10^{-3}$ to $10^{-4} \mathrm{M}$.) As expected, octopamine lowered the stimulus threshold for $A$. This result was obtained in all six experiments carried out with octopamine. Moreover, the average percentage decrease of $A$ 's threshold (18 \pm $5.8 \%$ SEM) was about the same as that of the LGs (12 $\pm 4.9 \%$ SEM), whose threshold was also tested in all but one of these experiments. 5-HT, however, did not consistently affect $A$ 's stimulus threshold. Of eight experiments, there was no change in $A$ 's threshold in three experiments, a small increase in three, and a decrease in two. The mean percentage change was $-2 \pm 3.8 \%$ SEM (threshold increases counted positive). By contrast, the threshold of the LGs rose in all of the experiments but one where the 5-HT (which oxidizes readily) had been in solution for $5 \mathrm{hr}$. Excluding this experiment, the mean rise of the LG threshold was $28 \pm 7.6 \%$ SEM.

\section{Discussion}

Because the LGs are the command neurons for one type of tailflip escape response (Wiersma, 1938; Wine and Krasne, 1972; Olson and Krasne, 1981), our results necessarily imply that 5-HT and octopamine have opposite modulatory effects upon this behavior; specifically, 5-HT decreases the probability of an LG response, whereas octopamine increases it.

The discovery of monoaminergic effects on this neurophysiologically well understood behavior promises further advances relevant to a number of important problems, such as the relationship between behavioral and neural modulation, the natural biological uses of central monoamines, the organization of monoaminergic systems, and the interactions of separate monoaminergic systems with each other and with non-monoaminergic systems in the mediation of behavior. Before the crayfish's LG system can begin to yield insights into these problems, however, several questions, discussed below, require answers.

Cellular actions mediating the monoamines' effects. Perhaps the most important question to be answered is whether the effects of 5-HT and octopamine on the LGs' responsiveness are in fact mediated by "modulatory" synaptic actions (cf. Kupfermann, 1979). Intracellular experiments on interneuron $\mathrm{A}$, along the lines of those previously used to examine other sorts of plasticity within the LG system (Zucker, 1972b; Bryan and Krasne, 1977a, b), may establish whether neuromodulatory actions underlie octopamine's effects. Further analysis of 5-HT's effects, however, must await localization of its site of action. The serotonergic effect is mostly confined to the disynaptic pathway to the LGs from the sensory afferents, but it seems to be absent at the synapse between the afferents and interneuron $\mathrm{A}$. We therefore presume that 5 -HT causes transmission to be depressed either at the synapse between sensory interneurons and the LGs or at the synapse between afferents and sensory interneurons other than $A$.

It is important to determine how widespread are 5HT's and octopamine's effects in the escape circuit because it is commonly believed that monoaminergic systems affect the nervous system's state rather broadly. That the monoamines must act on loci other than the afferent-to-sensory interneuron synapse can be inferred from 5-HT's apparent lack of effect upon A's threshold, and also from both monoamines' occasional effects upon 
the $\alpha$ component of the LG EPSP; but it remains to be seen whether 5-HT and octopamine have other, perhaps major effects upon motor elements in the LG system. Although we have no information on the effects of these two monoamines on the phasic motor system, their effects on the separate tonic motor system of the crayfish have been well studied (Livingstone et al., 1980; Kravitz et al., 1981). 5-HT has mixed effects but generally serves to enhance tonic flexion, whereas octopamine depresses tonic flexion and enhances tonic extension. At first glance these results might appear to be somewhat inconsistent with those reported here. However, the tonic and phasic motor systems do not necessarily function in parallel; in fact, tonic flexion is inhibited by LG-evoked phasic flexion of the abdomen (Kuwada and Wine, 1979). Hence, the contrapuntal effects of octopamine and 5-HT on the crayfish's IG escape response and on its posture may be viewed as synergistic, and may imply that these monoamines have widespread effects on the neural circuitry mediating escape behavior.

Finally, in several instances where monoamines are known to have neuromodulatory actions, "second messengers," e.g., cAMP, appear to mediate these actions (Bloom, 1975; Drummond et al., 1980; Enyeart, 1981; Kandel et al., 1981). Whether a second messenger mediates either the effect of 5-HT or that of octopamine on the LG escape reflex remains to be determined.

Natural delivery mode of the monoamines. Careful assays of lobster hemolymph indicate that the levels of 5$\mathrm{HT}$ and octopamine circulating in free form are less than $10^{-8} \mathrm{M}$, and it is likely that a similar result would hold true for the crayfish. This is too low a concentration to produce the previously described central effects of 5-HT and octopamine in these animals (Livingstone et al., 1980; Kravitz et al., 1981). The effects of 5-HT and octopamine on the LGs are present, but relatively small, at $10^{-6} \mathrm{M}$. Thus, although we have not yet determined threshold concentrations, it seems unlikely that these effects will persist at $10^{-8} \mathrm{M}$. This suggests that both the previously described central effects of the monoamines in lobsters and crayfish and those described here may be due not to their release into the blood, but, rather, to their release at central synapses (Kravitz et al., 1981; cf. Bailey et al., 1981 for related data). In support of this notion, an endogenous system of 5-HT-containing neurons has recently been discovered in the lobster's abdominal nerve cord (Beltz and Kravitz, 1983). Moreover, we have recently found monoaminergic-like terminals in the region of afferent terminals within the crayfish's sixth abdominal ganglion (unpublished data). Some of these terminals resemble those identified as octopaminergic and others, those identified as serotonergic in the lobster (Livingstone et al., 1981).

Behavioral significance of the monoamines' effects. We suspect that the effects described here mimic ones normally resulting from activity in central monoaminergic pathways whose function is to set the level of excitability for the escape response. Thus, 5 -HT might mediate the suppressive effects of restraint and feeding upon the escape response (Krasne and Wine, 1975; F. B. Krasne, unpublished data). Consistent with this hypothesis, transmission from sensory afferents to interneuron $A$ is not suppressed during either restraint-induced (Krasne and Wine, 1975) or feeding-induced (unpublished data) inhibition of the LG response. By analogy with Aplysia (Kandel et al., 1981), octopamine might be expected to mediate sensitization of the escape response. Behavioral sensitization of this response has yet to be demonstrated, but, spurred by the present results, we have begun attempts to do so. These or other natural roles for $5-\mathrm{HT}$ and octopamine in crayfish escape behavior remain to be established; however, if such roles can be found, the crayfish's escape response should constitute a valuable model system for studying the functions of monoamines in the central nervous system's mediation of behavior.

\section{References}

Bailey, C. H., R. D. Hawkins, M. C. Chen, and E. R. Kandel (1981) Interneurons involved in mediation and modulation of gill-withdrawal reflex in Aplysia. IV. Morphological basis of presynaptic facilitation. J. Neurophysiol. 45: 340-360.

Battelle, B. A., and E. A. Kravitz (1978) Targets of octopamine action in the lobster: Cyclic nucleotide changes and physiological effects in hemolymph, heart and exoskeletal muscle. J. Pharmacol. Exp. Ther. 205: 438-448.

Beltz, B. S., and E. A. Kravitz (1983) Mapping of serotoninlike immunoreactivity in the lobster nervous system. J. Neurosci. 3: 585-602.

Bloom, F. E. (1975) The role of cyclic nucleotides in central synaptic function. Rev. Physiol. Biochem. Pharmacol. 74: 1103.

Brown, S. K., and D. N. Sherwood (1981) Vascularization of the crayfish abdominal nerve cord. J. Comp. Physiol. 143: 93-101.

Bryan, J. S., and F. B. Krasne (1977a) Protection from habituation of the crayfish lateral giant fiber escape response. J. Physiol. (Lond.) 271: 351-368.

Bryan, J. S., and F. B. Krasne (1977b) Presynaptic inhibition: The mechanism of protection from habituation of the crayfish lateral giant escape response. J. Physiol. (Lond.) 271: 369-390.

Candy, D. J. (1978) The regulation of locust flight muscle metabolism by octopamine and other compounds. Insect Biochem. 8: 177-181.

Davis, M., D. I. Astrachan, and E. Kass (1980) Excitatory and inhibitory effects of serotonin on sensorimotor reactivity measured with acoustic startle. Science 209: 521-523.

Drummond, A. H., J. A. Benson, and I. B. Levitan (1980) Serotonin-induced hyperpolarization of an identified Aplysia neuron is mediated by cyclic AMP. Proc. Natl. Acad. Sci. U. S. A. 77: 5013-5017.

Dudel, J. (1965) Facilitatory effects of 5-hydroxytryptamine on the crayfish neuromuscular junction. Naunyn-Schmiedebergs Arch. Exp. Pathol. Pharmakol. 249: 515-528.

Enyeart, J. (1981) Cyclic AMP, 5-HT, and the modulation of transmitter release at the crayfish neuromuscular junction. J. Neurobiol. 12: 505-513.

Evans, P. D., and M. O'Shea (1978) The identification of an octopaminergic neuron and the modulation of a myogenic rhythm in the locust. J. Exp. Biol. 73: 235-260.

Gelperin, A. (1981) Synaptic modulation by identified serotonin neurons. In Serotonin Neurotransmission and Behavior, B. L. Jacobs and A. Gelperin, eds., pp. 288-304, MIT Press, Cambridge, MA.

Grundfest, H., and J. P. Reuben (1961) Neuromuscular synaptic activity in lobster. In Nervous Inhibition, E. Florey, ed., pp. 92-104, Pergamon Press, New York.

Horvitz, H. R., M. Chalfie, C. Trent, J. E. Sulston, and P. D. 
Evans (1982) Serotonin and octopamine in the nematode Caenorhabditis elegans. Science 216: 1012-1014.

Hoyle, G., and D. Dagan (1978) Physiological characteristics and reflex activation of DUM (octopaminergic) neurons of locust metathoracic ganglion. J. Neurobiol. 9: 59-79.

Jacobs, J. R., and H. L. Atwood (1981) Long term facilitation of tension in crustacean muscle and its modulation by temperature, activity and circulating amines. J. Comp. Physiol. 144: 335-343.

Kandel, E. R., M. Klein, C. H. Bailey, R. D. Hawkins, V. F. Castellucci, B. W. Lubit, and J. H. Schwartz (1981) Serotonin, cyclic AMP, and the modulation of the calcium current during behavioral arousal. In Serotonin Neurotransmission and Behavior, B. L. Jacobs and A. Gelperin, eds., pp. 211254, MIT Press, Cambridge, MA.

Krasne, F. B. (1969) Excitation and habituation of the crayfish escape reflex: The depolarizing response in lateral giant fibers of the isolated abdomen. J. Exp. Biol. 50: 29-46.

Krasne, F. B., and S. H. Lee (1977) Survival of functional synapses on crustacean neurons lacking cell bodies. Brain Res. 121: 43-57.

Krasne, F. B., and J. J. Wine (1975) Extrinsic modulation of crayfish escape behavior. J. Exp. Biol. 63: 433-450.

Krasne, F. B., and J. J. Wine (1977) Control of crayfish escape behavior. In Identified Neurons and Behavior of Arthropods, G. Hoyle, ed., pp. 275-292, Plenum Press, New York.

Kravitz, E. A., S. Glusman, M. S. Livingstone, and R. M. Harris-Warrick (1981) Serotonin and octopamine in the lobster nervous system: Mechanism of action at neuromuscular junctions and preliminary behavioral studies. In Serotonin Neurotransmission and Behavior, B. L. Jacobs and A. Gelperin, eds., pp. 189-210, MIT Press, Cambridge, MA.

Kupfermann, I. (1979) Modulatory actions of neurotransmitters. Annu. Rev. Ncurosci. 2: 447-465.

Kupfermann, I., and K. R. Weiss (1981) The role of serotonin in arousal of feeding behavior of Aplysia. In Serotonin Neurotransmission and Behavior, B. L. Jacobs and A. Gelperin, eds., pp. 255-287, MIT Press, Cambridge, MA.

Kuwada, J. Y., and J. J. Wine (1979) Crayfish escape behavior:
Commands for fast movement inhibit postural tone and reflexes, and prevent habituation of slow reflexes. J. Exp. Biol. 79: 205-224.

Livingstone, M. S., R. M. Harris-Warrick, and E. A. Kravitz (1980) Serotonin and octopamine produce opposite postures in lobsters. Science 208: 76-79.

Livingstone, M. S., S. F. Schaeffer, and E. A. Kravitz (1981) Biochemistry and ultrastructure of serotonergic nerve endings in the lobster: Serotonin and octopamine are contained in different nerve endings. J. Neurobiol. 12: 27-54.

Olson, G. C., and F. B. Krasne (1981) The crayfish lateral giants as command neurons for escape behavior. Brain Res. 214: $89-100$.

O'Shea, M., and P. D. Evans (1979) Potentiation of neuromuscular transmission by an octopaminergic neuron in the locust. J. Exp. Biol. 79: 169-190.

Ungerstedt, U. (1979) Central dopamine mechanisms and unconditioned behavior. In The Neurobiology of Dopamine, A. S. Horn, J. Korf, and B. H. C. Westerink, eds., pp. 577-596, Academic Press, Inc., New York.

Van Harreveld, A. (1936) A physiological solution for fresh water crustaceans. Proc. Soc. Exp. Biol. Med. 34: 428-432.

Wiersma, C. A. G. (1938) Function of the giant fibers of the central nervous system of the crayfish. Proc. Soc. Exp. Biol. Med. 38: 661-662.

Willard, A. L. (1981) Effects of serotonin on the generation of the motor program for swimming by the medicinal leech. J. Neurosci. 1: 936-944.

Wine, J. J., and F. B. Krasne (1972) The organization of escape behavior in the crayfish. J. Exp. Biol. 56:1-18.

Zucker, R. S. (1972a) Crayfish escape behavior and central synapses. I. Neural circuit exciting lateral giant fiber. J. Neurophysiol. 35: 599-620.

Zucker, R. S. (1972b) Crayfish escape behavior and central synapses. II. Physiological mechanisms underlying behavioral habituation. J. Neurophysiol. 35: 621-637.

Zucker, R. S. (1972c) Crayfish escape behavior and central synapses. III. Electrical junctions and dendritic spikes in fast flexor motoneurons. J. Neurophysiol. 35: 638-651. 